\title{
Preparation and properties evaluation of polyimide-matrix nanocomposites reinforced with glutamine functionalized multi-walled carbon nanotube
}

\author{
Edris Kianpour Kalchounaki ${ }^{1}$ Asadollah Farhadi ${ }^{2} \cdot{\text { Amin } \text { Zadehnazari }^{2}}^{2}$
}

Received: 16 February 2018 / Revised: 27 April 2018 / Accepted: 3 May 2018 /

Published online: 8 May 2018

(C) The Author(s) 2018

\begin{abstract}
L-glutamine-grafted multi-walled carbon carboxylic acid nanotube (denoted as L-Gl-MWCNT) and polyimide-matrix L-Gl/MWCNT (L-Gl-MWCNT/ $\mathrm{PI})$ were prepared by in situ polymerization and solution-blending processes. The functionalization process was one-pot, fast, and simple, and resulted in a high degree of functionalization as well as dispersibility in organic solvents. The resulting materials were characterized by Fourier transform infrared spectroscopy, thermogravimetric analysis, X-ray diffraction, high-resolution transmission electron microscopy, and field-emission scanning electron microscopy. According to the microscopic characterizations, the MWCNTs are homogeneously dispersed in the composites. The thermal stability of the films containing Gl-MWCNTs was improved as the nanotube content increased from 5 to $15 \mathrm{wt} \%$ due to the improved interfacial interaction between the PI matrix and surface-modified MWCNTs.
\end{abstract}

Keywords Gl-MWCNT/PI $\cdot$ TGA $\cdot$ XRD $\cdot$ TEM $\cdot$ FESEM

\section{Introduction}

High-performance/high-temperature polymers are characterized by their excellent balance of thermal and mechanical properties which makes them useful materials for engineering applications. Modification of the polymers physical properties as well as the implementation of new features in the polymer matrix was carried out by the addition of inorganic spherical nanoparticles to polymers. Over the past two

Asadollah Farhadi

farhadichem@put.ac.ir

1 Department of Chemical Engineering, Abadan Branch, Islamic Azad University, Abadan, Iran

2 Faculty of Science, Petroleum University of Technology, Ahvaz 61981-44471, Iran 
decades, materials and structures with geometric dimensions of less than $100 \mathrm{~nm}$ (nanomaterial) have gained more attraction for chemistry and pharmaceuticals, such as synthesis of organic compounds, health, medical, or food technology [1-10].

In 1991, existence of carbon nanotubes opened new advancement paths of the interdisciplinary sciences, because these compounds have a unique atomic structure and a very high aspect ratio [11]. The chemical and thermal stability, electronic properties, high tensile strength, flexibility, adsorption, and ultra-light weight are the main features of multi-walled carbon nanotubes (MWCNTs). These materials offer a plethora of application potential in many different branches of science [12-15]. Also, different ways of MWCNT synthesis due to its application in electromagnetic shielding (EMI), sensors, electrostatic charge dissipation (ESD), flame retardancy, wind turbine blades, photovoltaic packaging, electrically conducting cables were reported [16]. However, carbon nanotubes (CNTs) due to these properties are one of the best aforesaid catalyst supports and be making them ideal reinforcing fibers in nanocomposites [12]. These compounds have been used in the production of polymer nanocomposites [13]. Due to the dimensions of carbon nanofibers (CNFs) (between 50 and $200 \mathrm{~nm}$ ) [17], these compounds are similar to those of singlewalled carbon nanotubes (SWCNTs) and multi-walled carbon nanotubes (MWCNTs) [18].

In this contribution in order to achieve fine dispersion of carboxyl-functionalized multi-walled CNTs (MWCNTs), we attempted to prepare MWCNT/PI composites using in situ polymerization approach (i.e., solution-casting followed by subsequent imidization).

\section{Experimental}

\section{Materials}

All materials and solvents were purchased either from Aldrich Chemical Co. (Milwaukee, WI), Merck or Fluka (Germany).

\section{Characterization}

Fourier transform infrared (FTIR) spectra of the samples were recorded on a Bomem MB-Series 1998 FTIR spectrophotometer (Quebec, Canada) at a resolution of $4 \mathrm{~cm}^{-1}$. The samples were examined as a disk, grinded together with potassium bromide (KBr) salt. The morphology of the MWCNTs and the dispersion morphology of the MWCNTs on the PI matrix were observed using field-emission scanning electron microscopy (FESEM). The images were taken at $15 \mathrm{kV}$ using a HITACHI S-4160 instrument (Tokyo, Japan). Transmission electron microscopy (TEM) images were obtained using a Philips CM 120 microscope (Netherlands) with an accelerating voltage of $100 \mathrm{kV}$. For TEM studies, ultra-thin sections $(30-80 \mathrm{~nm})$ of the composites were prepared using Leica Ultramicrotome. Branson S3200 $(50 \mathrm{kHz}$, 


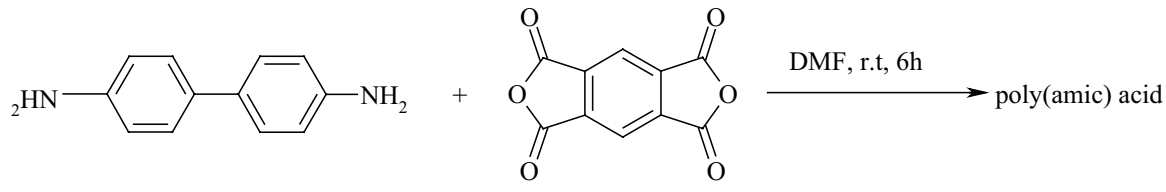

Scheme 1 Synthesis of poly(amic acid) (PAA) at room temperature
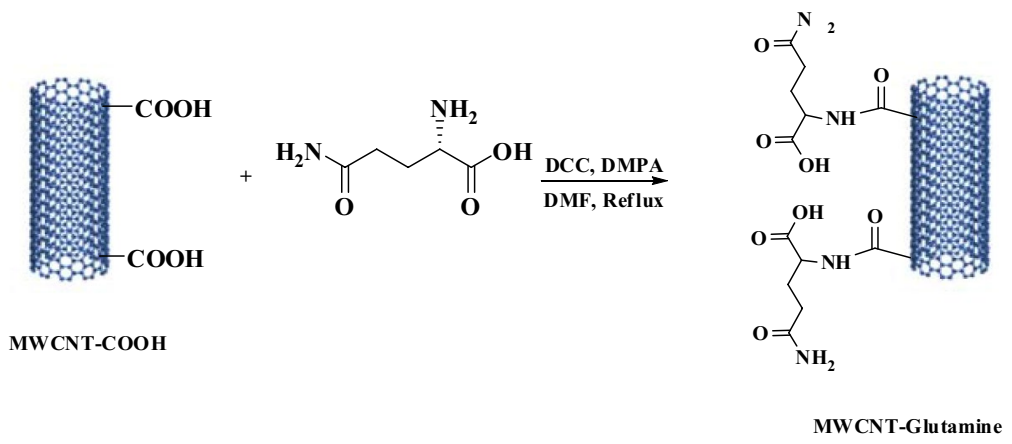

Scheme 2 Synthesis of MWCNT-glutamine under reflux condition

$150 \mathrm{~W}$ ) ultrasonic bath (Americas Headquarters 41 EagleRoad, Danbury CT 06810, USA) was used for better dispersion of MWCNTs.

\section{Synthesis of polyamic acid (PAA)}

$0.3 \mathrm{~g}$ of benzidine diamine and $0.355 \mathrm{~g}$ of 1,2,4,5-benzene tetracarboxylic dianhydride were mixed in $5 \mathrm{~mL} N, N$-dimethyl formamide (DMF) as solvent at room temperature. The reaction mixture was stirred for $6 \mathrm{~h}$ by a mechanical stirrer at this temperature (Scheme 1).

\section{Synthesis of Gl-MWCNTs}

$0.6 \mathrm{~g}$ of MWCNTs- $\mathrm{CO}_{2} \mathrm{H}, 6 \mathrm{~g}$ of L-glutamine, $3 \mathrm{~g} \mathrm{~N}, \mathrm{~N}$-dicyclohexylcarbodiimide (DCC), and $1.8 \mathrm{~g}$ 4-dimethyl aminopyridine (DMAP) were mixed in the $60 \mathrm{~mL}$ DMF. The mixture was placed in a beaker and stirred under ultrasonication for $2 \mathrm{~h}$ at room temperature to reach a homogenous suspension. Then this mixture was refluxed at $100{ }^{\circ} \mathrm{C}$ for 4 days. The crude mixture was washed with ethanol and distilled water. At the end, the product was kept at $80^{\circ} \mathrm{C}$ for $8 \mathrm{~h}$. The black product was characterized by FTIR, TG, FESEM, TEM, and XRD spectra (Scheme 2). 


\section{Synthesis of L-Gl-MWCNT/Polyimide (L-Gl-MWCNT/PI)}

The L-Gl-MWCNT/PI composite films were prepared by solution-casting method under three steps.

1. In a typical process, a certain amount of benzidine diamine and 1,2,4,5-benzene tetracarboxylic dianhydride was dissolved in DMF completely with stirring until the PAA solution was obtained.

2. Separately, $3 \mathrm{~mL}$ of DMF and the appropriate amount (from 5 to $15 \mathrm{wt} \%$ ) of L-Gl-MWCNT were placed in a beaker and stirred under ultrasonication for $1 \mathrm{~h}$ at room temperature to reach a homogenous suspension. This beaker was added to the PAA mixture prepared above and further stirred for $12 \mathrm{~h}$.

3. The composite films were prepared by coating $3 \mathrm{~mL}$ of the final mixture solution on a piece of $50 \mathrm{~mm} \times 50 \mathrm{~mm}$ glass slide. The resultant tack-free films were dried in an oven in air atmosphere. The imidazation process was occurred in a heating program as follow: ramping from room temperature to $50{ }^{\circ} \mathrm{C}$ in $30 \mathrm{~min}$, keeping at $50{ }^{\circ} \mathrm{C}$ for $90 \mathrm{~min}$, then ramping from 50 to $80^{\circ} \mathrm{C}$ in $1 \mathrm{~h}$, and then from 80 to $100{ }^{\circ} \mathrm{C}$ in $2 \mathrm{~h}$, keeping at $100{ }^{\circ} \mathrm{C}$ for $1 \mathrm{~h}$. The product was synthesized by the following method (Scheme 3).

\section{Results and discussion}

The FTIR spectrum was used to analyze the chemical structure of samples. The FTIR spectra of the L-Gl-MWCNT (compound a), neat PI (compound b), L-GlMWCNT/PI with $5 \mathrm{wt} \%$ (compound c), $10 \mathrm{wt} \%$ (compound d), and $15 \mathrm{wt} \%$ (compound e) carbon nanotube are shown in Fig. 1.

FTIR (KBr) (compound a): 3238-4000 $\left(\mathrm{CO}_{2} \mathrm{H}\right.$, broad and strong), 3928, 3413, 3238 (NH, weak), 2922, 2853 ( $\mathrm{C}-\mathrm{H}$, aliphatic, weak), $1617.76(\mathrm{C}=\mathrm{O}$, strong), 1454.73 ( $\mathrm{C}=\mathrm{O}$, NHCO, strong), $1384.05(\mathrm{C}=\mathrm{C}), 1118 \mathrm{~cm}^{-1}$ (C-O, weak).

FTIR (KBr) (compound b): 3470.33-2500 ( $\mathrm{CO}_{2} \mathrm{H}$, broad and strong), 3470.33, $3413.01\left(\mathrm{NH}\right.$, weak), $3040.29(\mathrm{C}-\mathrm{H}$ aromatic $), 1722.96\left(\mathrm{C}=\mathrm{O}, \mathrm{CO}_{2} \mathrm{H}\right.$, strong) 1662.60 ( $\mathrm{C}=\mathrm{O}$, NHCO, strong), $1500.81(\mathrm{C}=\mathrm{C}), 1108.95 \mathrm{~cm}^{-1}$ (C-O, weak).

FTIR (KBr) (compound c): 3927.32, 3727.39 (N-H, very weak shoulder), 3500-2859.58 $\left(\mathrm{CO}_{2} \mathrm{H}\right.$, broad and strong), 3035.31 (C-H aromatic, weak), 2922.67, $2859.58\left(\mathrm{C}-\mathrm{H}\right.$, aliphatic, weak), $1718.33\left(\mathrm{C}=\mathrm{O},\left(\mathrm{CO}_{2} \mathrm{H}\right)\right.$, strong $), 1643.89(\mathrm{C}=\mathrm{O}$, CONH, strong), 1498.67, $1381.63(\mathrm{C}=\mathrm{C}), 1104.92 \mathrm{~cm}^{-1}$ (C-O, weak).

FTIR (KBr) (compound d): $3929.33(\mathrm{~N}-\mathrm{H}$, very weak shoulder), 3548-32.36.95 $\left(\mathrm{CO}_{2} \mathrm{H}\right.$, broad and strong), 3548.84, $3412.45(\mathrm{~N}-\mathrm{H}$, very weak shoulder $), 3037.86$ (C-H, aromatic, weak), 2923.86, 2855.52 (C-H, aliphatic, weak), $1776.90(\mathrm{C}=\mathrm{O}$, $\left(\mathrm{CO}_{2} \mathrm{H}\right)$, strong), $1721.25(\mathrm{C}=\mathrm{O}, \mathrm{CONH}$, strong), $1616.86(\mathrm{C}=\mathrm{O}, \mathrm{CONH}$, strong), 1498.28, 1377.75 ( $\mathrm{C}=\mathrm{C}$, strong), $1102.96 \mathrm{~cm}^{-1}$ (C-O, weak).

FTIR (KBr) (compound e): $3930.30(\mathrm{~N}-\mathrm{H}$, very weak shoulder), 3550-2923 $\left(\mathrm{CO}_{2} \mathrm{H}\right.$, broad and strong), 3550.36, $3412.66(\mathrm{~N}-\mathrm{H}$, very weak shoulder $), 3038.01$ (C-H, aromatic, weak), $2923.00\left(\mathrm{C}-\mathrm{H}\right.$, aliphatic, weak), $1719.95\left(\mathrm{C}=\mathrm{O},\left(\mathrm{CO}_{2} \mathrm{H}\right)\right.$, 
Step 1<smiles>Nc1ccc(-c2ccc(N)cc2)cc1</smiles><smiles>CC(C)(C)Nc1ccc(-c2ccc(NC(=O)c3cc(C(=O)O)c(C(=O)C(C)(C)C)cc3C(=O)O)cc2)cc1</smiles>

Step 2

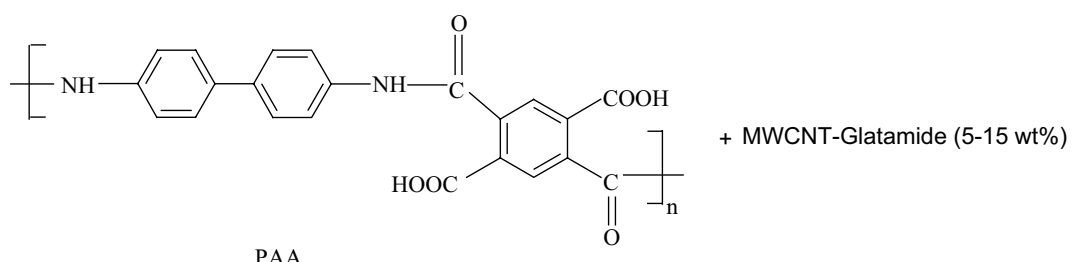<smiles>CC(C)(C)Nc1ccc(-c2ccc(-n3c(=O)c4cc5c(=O)n(C(C)(C)C)c(=O)c5cc4c3=O)cc2)cc1</smiles>

Scheme 3 Synthesis of PAA and MWCNT-glutamine/PI composite

strong), 1616.86 (C=O, $\mathrm{CONH}$, strong $), 1498.37,1378.01 \quad(\mathrm{C}=\mathrm{C}$, strong $)$, $1106.30 \mathrm{~cm}^{-1}$ (C-O, weak).

Due to the presence of a carboxylic acid group on MWCNT- $\mathrm{CO}_{2} \mathrm{H}$, all of the spectra of the FTIR, this group has in strong bonds with their absorption on $2500-3500 \mathrm{~cm}^{-1}$. The spectrum of PAA (compound a) show that free $\mathrm{OH}$ stretching of $\mathrm{CO}_{2} \mathrm{H}$ group is very broad relative to other compounds because the carboxylic acid group is located next to the amide group and can form a hydrogen bond. The intense peak near $3100-3500 \mathrm{~cm}^{-1}$ may be attributed to $\mathrm{NH}$ stretching 


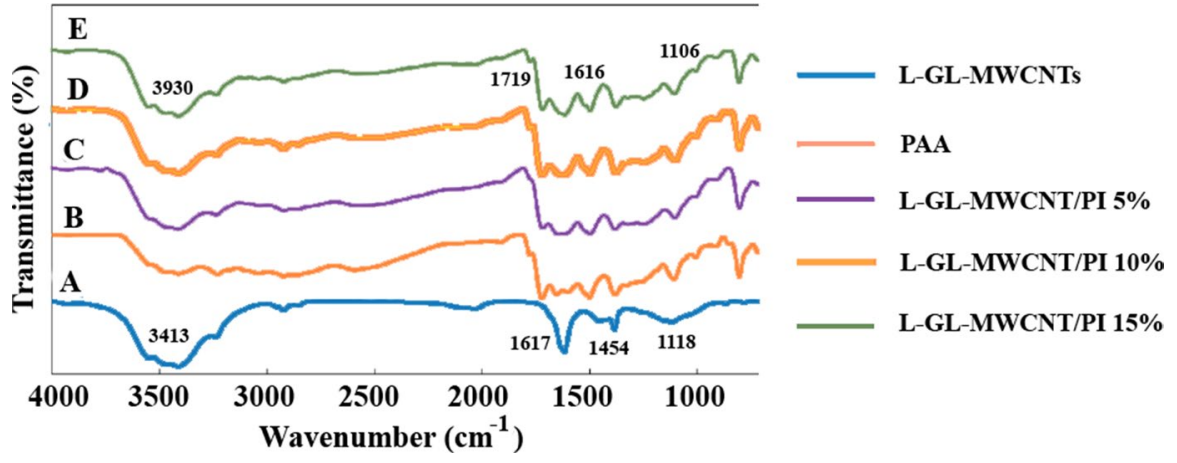

Fig. 1 FTIR spectra of A compound $\mathbf{a}, \mathbf{B}$ compound $\mathbf{b}, \mathbf{C}$ compound $\mathbf{c}, \mathbf{D}$ compound $\mathbf{d}, \mathbf{E}$ compound $\mathbf{e}$

groups, which are no longer seen in the blends spectra, because their signals are overlapped by the stronger $\mathrm{OH}$ stretching band. Compound $\mathrm{b}$ has asymmetric structure; however, two peaks of the amine group are shown $(\nu=3470.33$, $\left.3413.01 \mathrm{~cm}^{-1}\right)$.

Consequently, these data show that the imidazation process was not completed in a heating program.

The microstructure of the PAA and PI composites were studied by FESEM and TEM. Typical FESEM images of the PAA are presented in Fig. 2a. The neat PAA copolymer showed a spongy morphology. FESEM observation revealed that the PAA particles were self-organized into nanopatterns. As shown, the average diameter of polymeric particles was about less than $100 \mathrm{~nm}$ in more cases. By dispersing L-Gl-MWCNTs into the extremely diluted solution, the MWCNTs were observed to be several nanometers in diameter and several microns in length. FESEM observation (Fig. 2b-d) shows a fine and homogeneous dispersion of L-Gl-MWCNTs throughout PI matrix, as an example, for the fractured surface of the composite film containing $5 \mathrm{wt} \%$ of MWCNTs, and no aggregation of MWCNTs is observed. In addition, an intimate adhesion of MWCNTs with the matrix, indicating good wettability between them, is clearly observed.

The morphology and tubular structure of MWCNT-COOH, L-Gl-MWCNT, and the composite containing $10 \mathrm{wt} \%$ of L-Gl-MWCNT were also observed using TEM. As shown in Fig. 3 (top), the oxidation and amidation of MWCNTs did not deteriorate the structural integrity of MWCNTs. The sidewalls of MWCNTs-COOH were comparatively smooth and clean (Fig. 3, top), while they became rough after functionalization (Fig. 3, down). Compared to Fig. 3 (top, down) shows that the following amidation did not damage the CNTs. L-Gl-MWCNT specimens similarly were seen to have a high aspect ratio. Figure 4, L-Gl-MWCNT/PI composite $10 \mathrm{wt} \%$, shows that there was no observed deterioration in the homogeneously dispersed L-Gl-MWCNTs. It was clearly shown that the homogeneous behavior of MWCNT raised from the miscibility of the MWCNT within the PI chains is due to the strong interfacial interactions (non-covalent and hydrogen bonds) as well as the chemical compatibility between the PI matrix and the functionalized MWCNTs. 

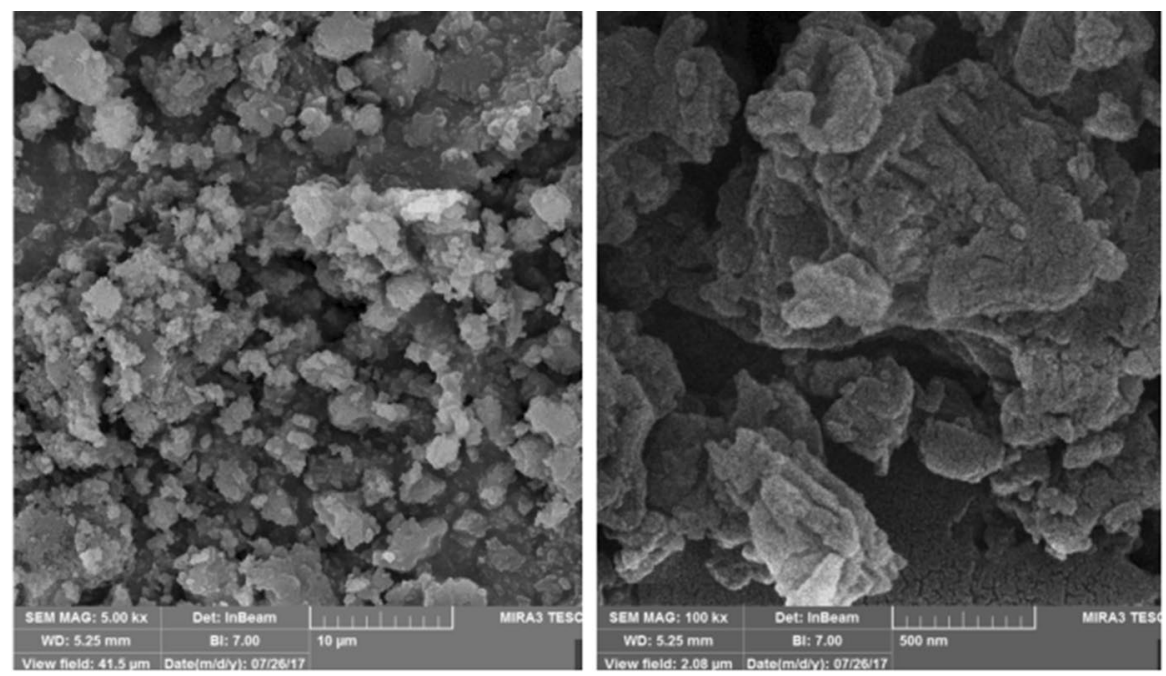

A
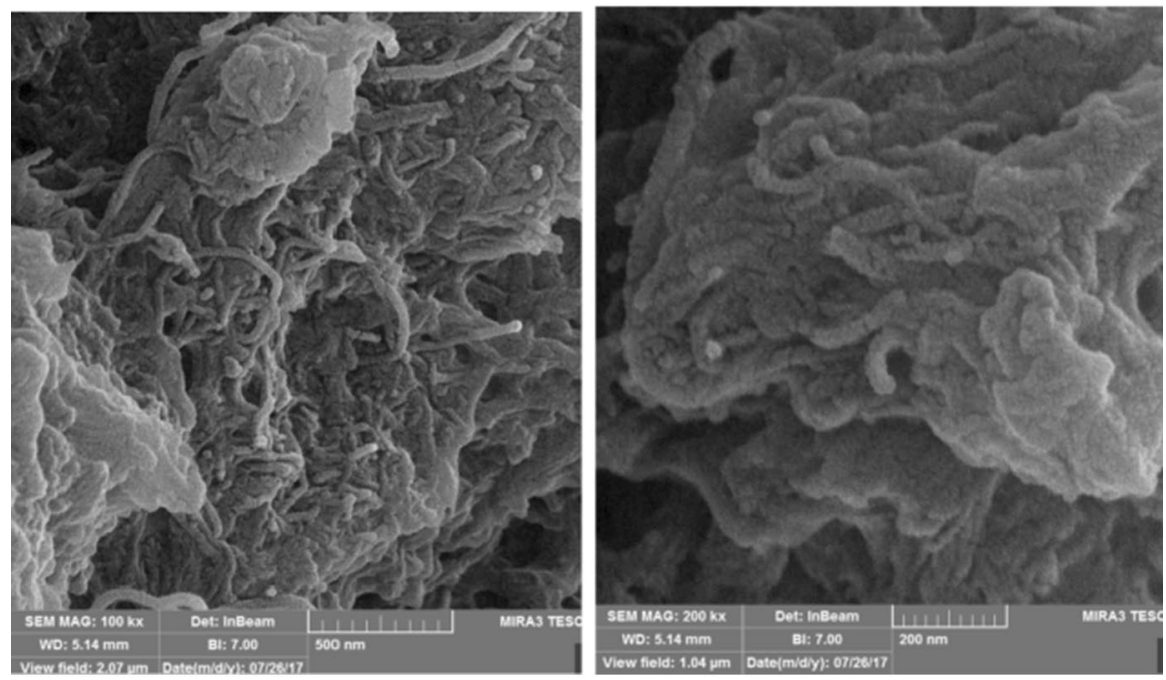

B

Fig. 2 Typical FESEM micrographs of a PAA and the fracture surface of the composites containing, $\mathbf{b}$ $5 \mathrm{wt} \%$, c $10 \mathrm{wt} \%$, and $\mathbf{d} 15 \mathrm{wt} \%$ of L-Gl-MWCNT

There are a handful of other characterization techniques of XRD elemental mapping for differentiating of L-Gl-MWCNT and PI compounds (Fig. 5).

The prominent peak about can be attributed to reflection of carbon.

The XRD pattern of L-Gl-MWCNT, as shown in Fig. 5a, shows a strong intensity at $2 \theta=11.5^{\circ}, 18^{\circ}, 20.5^{\circ}, 22.5^{\circ}, 266^{\circ}$ and a weak intensity at $44^{\circ}$ of the graphite crystal structure [19]. Meanwhile, for polyimide samples, the crystalline 

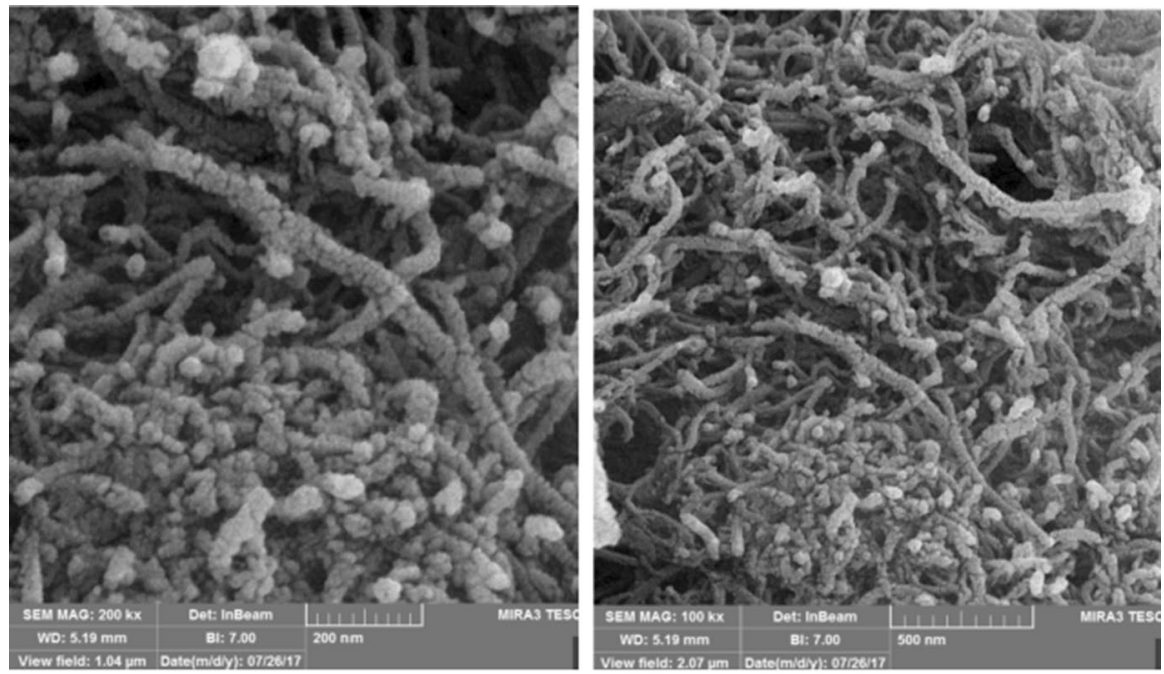

C
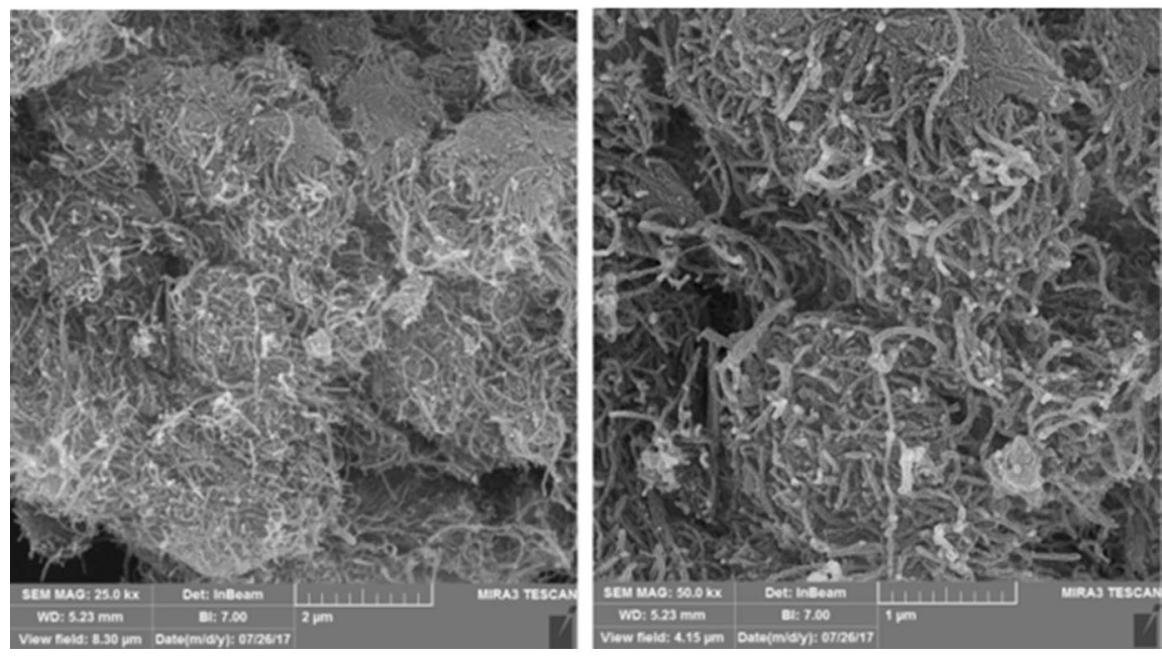

D

Fig. 2 (continued)

peaks appeared at $2 \theta=10.5^{\circ}, 20.5^{\circ}, 24.5^{\circ}, 26^{\circ}$ and $43^{\circ}$ [19]. The XRD pattern of the polymer nanocomposite with $5 \%$ carbon nanotube is shown in Fig. 5c. In this sample, the crystalline peaks appeared at $2 \theta=20.1^{\circ}, 24^{\circ}, 26.5^{\circ}$ and $43.5^{\circ}$ corresponding to reflections of PI [19].

At the same composite with $10 \mathrm{wt} \%$ carbon nanotube, the crystalline peaks appeared at $2 \theta=20.5^{\circ}, 25.5^{\circ}$, and $43^{\circ}$ (Fig. 5d). Furthermore, in the sample $\mathrm{E}$ with the same composite but $15 \mathrm{wt} \%$ carbon nanotube, the crystalline peaks appeared at $2 \theta=20^{\circ}, 26^{\circ}$ and $43.5^{\circ}$ (Fig. 5e). 

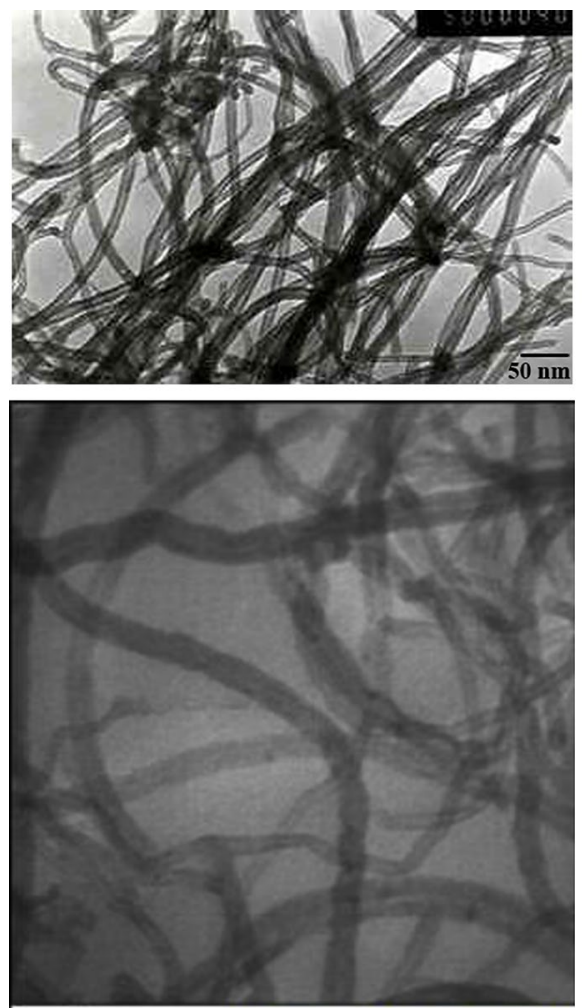

$60 \mathrm{~nm}$

$\mathrm{Mag}=60.000 \mathrm{KX}$ DayPetronic Ce

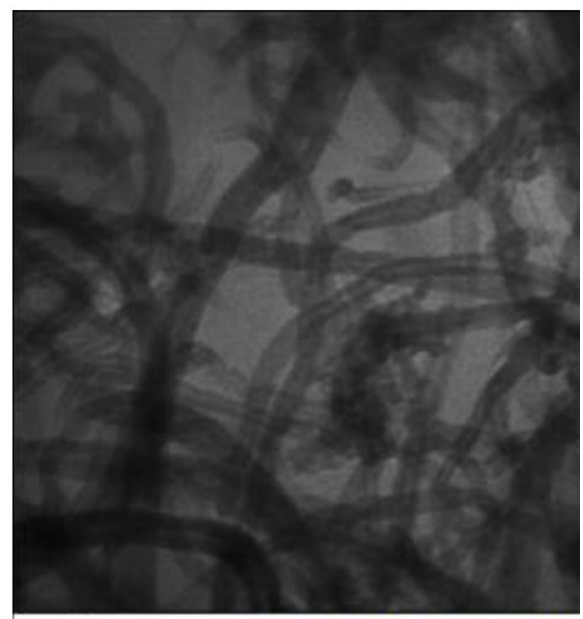

$60 \mathrm{~nm} \quad$ Mag $=60.000 \mathrm{KX} \quad$ DayPetronic Co

Fig. 3 TEM images of MWCNT-COOH (top) and L-Gl-MWCNT (down)

\section{Thermogravimeric analysis (TGA)}

TGA performed on numerous of polymer nanocomposite. These data show that many polymers filled with montmorillonite and carbon nanotubes exhibited improved thermal stability [20]. Therefore, in this study, the thermal behavior of MWCNTs- $\mathrm{CO}_{2} \mathrm{H}$, L-Gl-MWCNT, PAA, and L-Gl-MWCNT/PI was studied using TGA technique. The result of thermograms is shown in Fig. 6. However, the data obtained from them are reported in Table 1.

L-Gl-MWCNT has a stable weight loss before of $200{ }^{\circ} \mathrm{C}$ in TGA under nitrogen atmosphere. It is related to the decomposition of organic moieties on the surface of a nanotube wall. However, 5\% weight loss for carboxylated CNT is around $250{ }^{\circ} \mathrm{C}$, and the weight loss was less than $500{ }^{\circ} \mathrm{C}$, probably due to the decomposition temperature $\left(T_{\mathrm{d}}\right)$ of the amino acid group is in the range of $250-500{ }^{\circ} \mathrm{C}$.

The TGA of the PAA exhibited the good thermal stability; the 5 and $10 \%$ of weight loss was 132 and $177{ }^{\circ} \mathrm{C}$, respectively, and for the L-Gl-MWCNT/PI composites, these decomposition temperatures shifted toward higher temperatures as 

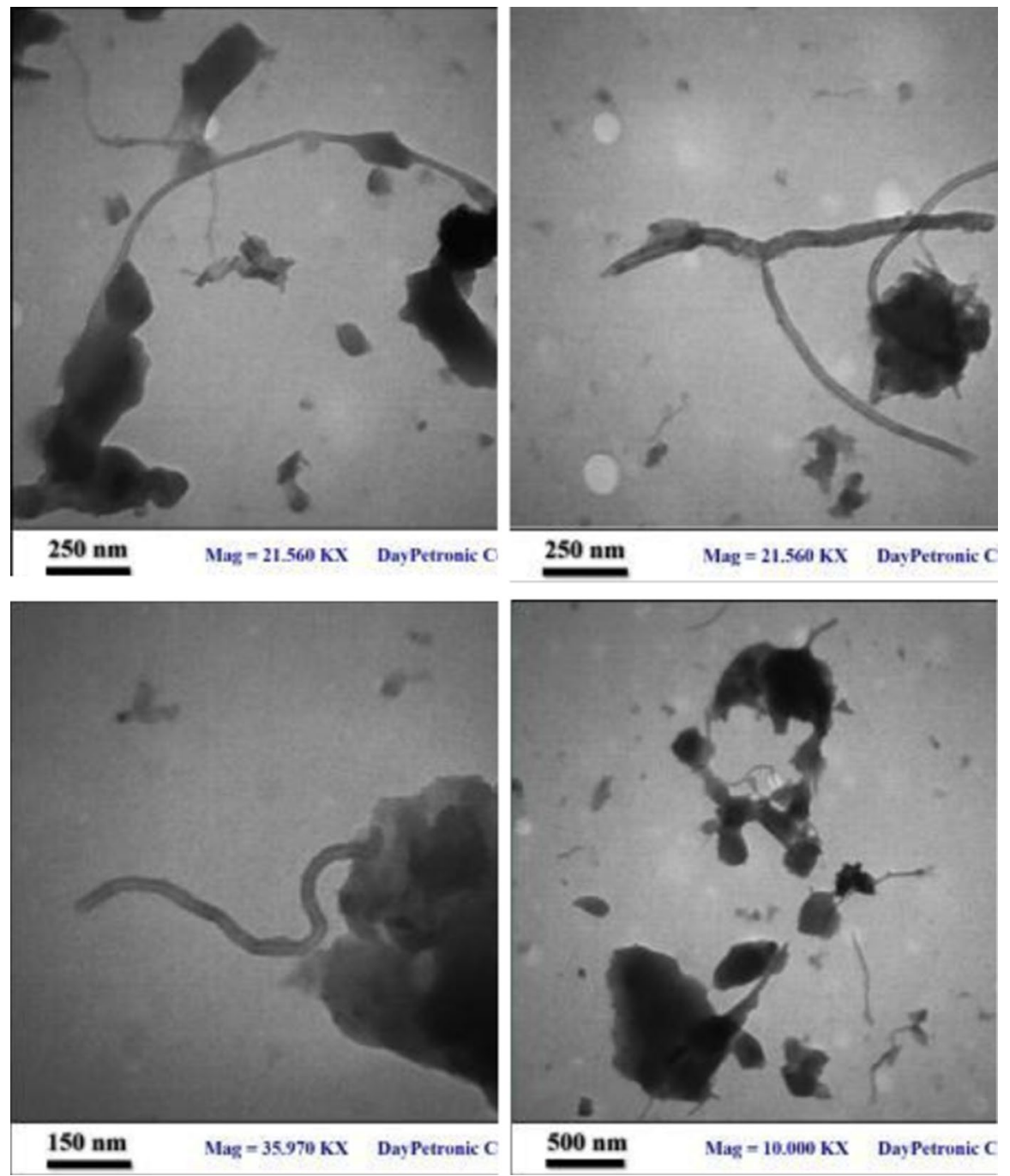

Fig. 4 TEM microphotographs of L-Gl-MWCNT/PI composite $10 \mathrm{wt} \%$

the amount of L-Gl-MWCNT was increased (Table 1). In Fig. 6, the onset of $T_{\mathrm{d}}$ of the L-Gl/MWCNT/PI composites was higher than that of pristine PI, and it shifted toward higher temperatures as the amount of L-Gl/MWCNT was increased. The $T_{\mathrm{d}}$ of pristine PAA and the composites with increasing amount of L-G1/MWCNT such as $0,5,10$, and $15 \%$ were $105,136,139$, and $147^{\circ} \mathrm{C}$, respectively. The $\mathrm{T}_{\mathrm{d}}$ of the composite with $15 \mathrm{wt} \% \mathrm{~L}-\mathrm{Gl} / \mathrm{MWCNT}$ exhibited a $T_{\mathrm{d}} 42{ }^{\circ} \mathrm{C}$ higher than that of pristine polymer. 


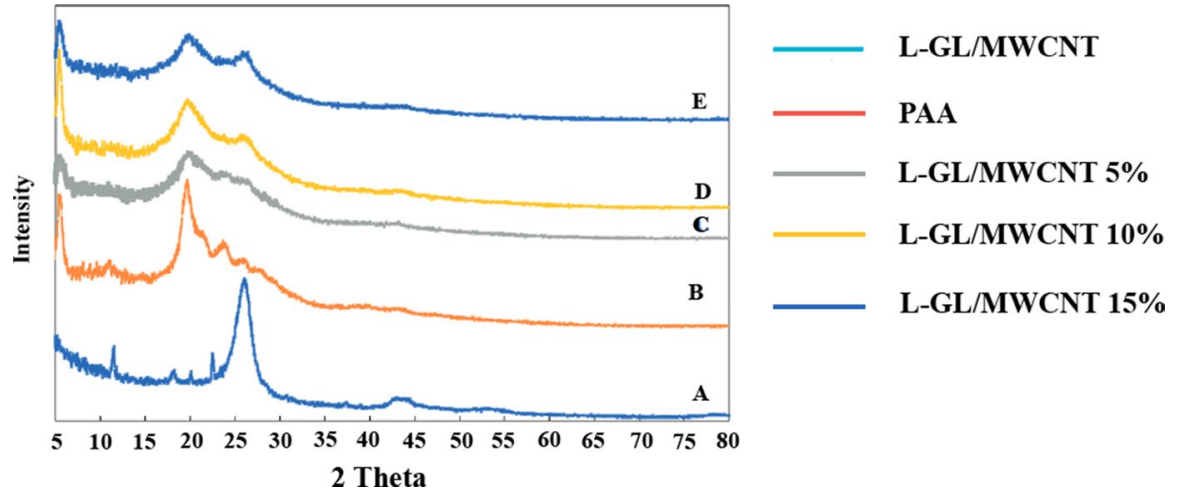

Fig. 5 XRD patterns of a L-Gl/MWCNT, b Neat PI, c L-Gl/MWCNT/PI 5 wt\%, d L-Gl/MWCNT/PI $10 \mathrm{wt} \%, \mathbf{e} \mathrm{L}-\mathrm{Gl} / \mathrm{MWCNT} / \mathrm{PI} 15 \mathrm{wt} \%$ of L-Gl/MWCNT

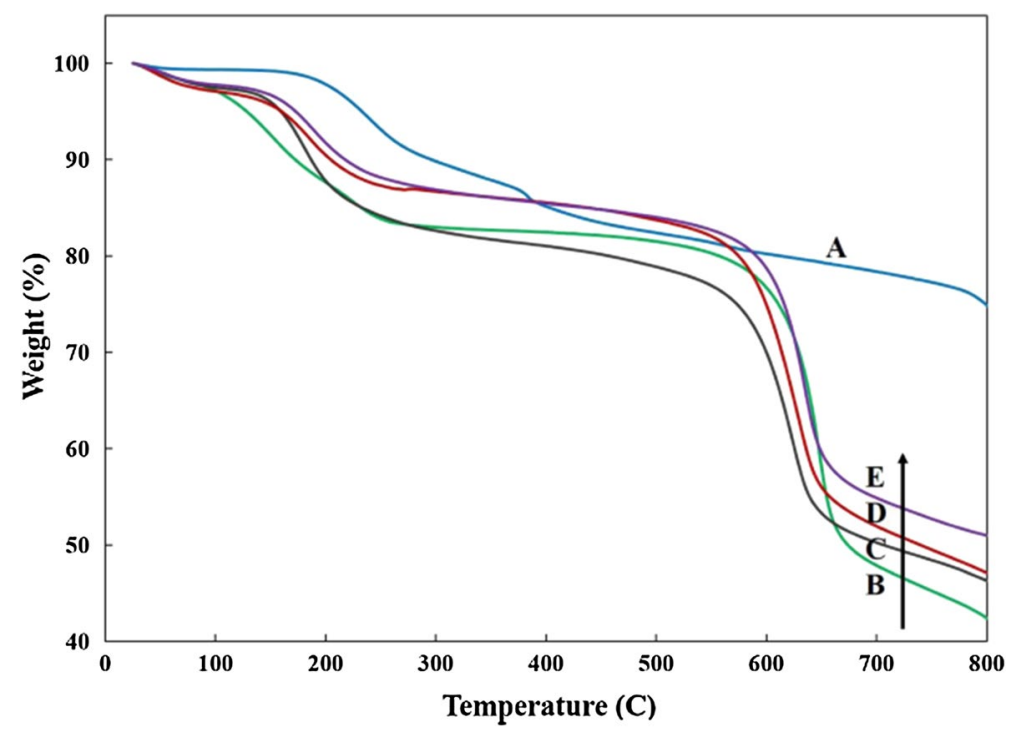

Fig. 6 TGA thermograms of a L-Gl-MWCNT, b PAA and L-Gl-MWCNT/PI composites with $\mathbf{c} 5 \%$, d $10 \%$, and $\mathbf{e} 15 \%$ of $\mathrm{L}-\mathrm{Gl} / \mathrm{MWCNT}$ content by weight

By comparing the obtained results from thermal testing with some of the previously reported L-G1/MWCNT/PI composites [21-23], it can be concluded that the thermal and mechanical stability of the prepared composited was significantly improved. The end temperature of decomposition was also increasing. The highest thermal decomposition temperature of $650{ }^{\circ} \mathrm{C}$ can be achieved. Therefore, it could be verified that a small amount of MWCNT acted as effective thermal degradation resistant reinforcement in the PI matrix, increasing the thermal stability of the L-Gl-MWCNT/PI composites. 
Table 1 Thermal properties of PAA, L-Gl/MWCNT and L-Gl/MWCNT/PI composites

\begin{tabular}{lllllll}
\hline Code & Onset $T_{\mathrm{d}}\left({ }^{\circ} \mathrm{C}\right)$ & $T_{\mathrm{d} 5}\left({ }^{\circ} \mathrm{C}\right)$ & $T_{\mathrm{d} 10}\left({ }^{\circ} \mathrm{C}\right)$ & $\mathrm{CR}(\%)$ & LOI $(\%)$ & $\Delta H_{\text {comb }}(\mathrm{kJ} / \mathrm{g})$ \\
\hline MWCNT & 400 & 407 & 761 & 89 & 53.1 & 15.1 \\
Net PAA & 105 & 132 & 177 & 42 & 34.3 & 23.3 \\
L-Gl/MWCNT & 224 & 236 & 307 & 75 & 47.5 & 16.8 \\
L-Gl/MWCNT/PI 5\% & 136 & 162 & 189 & 46 & 35.9 & 22.3 \\
L-Gl/MWCNT/PI 10\% & 139 & 164 & 209 & 47 & 36.3 & 22.0 \\
L-Gl/MWCNT/PI 15\% & 147 & 177 & 223 & 51 & 37.9 & 21.1 \\
\hline
\end{tabular}

Temperature at which 5 and $10 \%$ weight loss was recorded by TGA at a heating rate of $10{ }^{\circ} \mathrm{C} \mathrm{min}-1$ in a nitrogen atmosphere

According to Van Krevelen [24], there is a linear relationship between limiting oxygen index (LOI) and char residue $(\mathrm{CR})$ or char yield of polymers according to the following equation (Eq. 1):

$$
\mathrm{LOI}=17.5+0.4 \mathrm{CR}
$$

From this equation, PI, L-Gl-MWCNT, and L-Gl-MWCNT/PI containing 5, 10, and $15 \mathrm{wt} \%$ had LOI values of 34.3, 47.5, 35.9, 36.3, and 37.9, respectively. On the basis of the LOI values, such materials can be classified as self-extinguishing materials. According to Johnson [25], the LOI values of a lot of material scan also be rationally well predicted by the expression (Eq. 2):

$$
\mathrm{LOI}=8000 / \Delta H_{\mathrm{comb}}
$$

where $\Delta H_{\text {comb }}$ is the specific heat of combustion in $\mathrm{J} / \mathrm{g}$. So, in the case of PI, L-Gl/ MWCNT and L-Gl-MWCNT/PI composites (5, 10, and $15 \mathrm{wt} \%), \Delta H_{\text {comb }}$ is 15.1, $23.3,16.8,22.3,22.0$ and $21.1 \mathrm{~kJ} / \mathrm{g}$, respectively.

\section{Conclusion}

In this study, L-Gl-MWCNT/PI nanocomposites (from 5 to $15 \mathrm{wt} \%$ ) have been successfully synthesized in high purity from readily available reagents. In the next step, new classes of the L-Gl-MWCNT and L-Gl-MWCNT/PI hybrid composite films have been fabricated by in situ polymerization and solution-blending processes. CNTs remained structurally intact during preparation process and under subsequent workup conditions. A homogeneous dispersion of MWCNTs in the L-glutamine matrix was visually confirmed by detailed microscopic observations. The presence of L-Gl-MWCNT in the polymer matrix increased the thermal stability and reduced the thermal deformation of the polymer. The thermal stiffness effect of the composites was clearly demonstrated. The approach can be broadly used for the fabrication of the composites with better alignment and much higher volume fraction of nanofillers. By comparing the obtained results from thermal testing with some of the previously reported CNT/PI composites [21-23], it can be concluded that the thermal 
stability of the prepared composited was significantly improved. This study confirmed that the thermal and morphological properties of the composites are strongly dependent on the uniform dispersion of CNTs and the interactions between CNT and PI, which can be improved by using of modified CNT and the introduction of several functional groups in the polymer's chains.

Acknowledgements We are grateful to the Research Affairs Division of Petroleum University of Technology (PUT), Ahwaz Faculty of Petroleum Engineering for the financial support.

Open Access This article is distributed under the terms of the Creative Commons Attribution 4.0 International License (http://creativecommons.org/licenses/by/4.0/), which permits unrestricted use, distribution, and reproduction in any medium, provided you give appropriate credit to the original author(s) and the source, provide a link to the Creative Commons license, and indicate if changes were made.

\section{References}

1. Choi JH, Oh SB, Chang J, Il Kim, Ha CS, Kim BG, Han JH, Joo SW, Kim GH, Paik HJ (2005) Graft polymerization of styrene from single-walled carbon nanotube using atom transfer radical polymerization. Polym Bull 55:173-179

2. Andreea IB, Camelia H, Mihaela IA, Iuliana S, Dumitru P (2015) Polyimide precursor pattern induced by banded liquid crystal matrix: effect of dianhydride moieties flexibility. J Mater Sci 50:1358-1369

3. Lee CH, Liu JY, Chen SL, Wang YZ (2007) Miscibility and properties of acid-treated multi-walled carbon nanotubes/polyurethane nanocomposites 39:138-146

4. Faghihi K, Shabanian M, Hajibeygi M, Mohammadi Y (2010) Synthesis and properties of new thermally stable and optically active organosoluble poly(ether-amide-imide)s containing bicyclo segment in the main chain. J Appl Polym Sci 117:1184-1192

5. Hajibeygi M, Shabanian M, Moghanian H, Khonakdar HA, Haubler L (2015) Development of one-step synthesized LDH reinforced multifunctional poly(amide-imide) matrix containing xanthene rings: study on thermal stability and flame retardancy. RSC Adv 5:53726-53735

6. Shanmugharaj AM, Choi WS, Ryu SH (2011) Physical properties of phenol-anchored multiwall carbon nanotube/epoxy nanocomposite. Polym Bull 67:1721-1730

7. Asadinezhad A, Khonakdar HA, Scheffler C, Wagenknecht U, Heinrich G (2013) Poly(ethylene succinate)/single-walled carbon nanotube composites: a study on crystallization. Polym Bull 70:3463-3474

8. Farhadi A, Takassi MA, Dayer M (2011) Investigation of the interaction of ethyl acetoacetate with nano alumina particle as Lewis acid in acetonitrile solvent. Front Chem China 6:248-252

9. Shabanian M, Mirzakhanian Z, Moghanian H, Hajibeygi M, Salimi H, Khonakdar HA (2017) Thermal, combustion and optical properties of new polyimide/ODA-functionalized $\mathrm{Fe}_{3} \mathrm{O}_{4}$ nanocomposites containing xanthene and amide groups. J Therm Anal Calorim 129:147-159

10. Farhadi A, Takassi MA, Hejaz L (2013) Study of the synthesis of some Biginelli-type products catalyst by nano- $\mathrm{ZrO}_{2}$. Z Naturforsch B 68:51-56

11. Iijima S (1991) Helical microtubules of graphitic carbon. Nature 354:56-58

12. Frank S, Poncheral P, Wang ZL, Heer WA (1998) Carbon nanotube quantum resistors. Science 280:1744-1746

13. Ajayan PM, Stephan O, Colliex C, Trauth D (1994) Aligned carbon nanotube arrays formed by cutting a polymer resin-nanotube composite. Science 265:1212-1214

14. Bower C, Rosen R, Jin L, Han J, Zhou O (1999) Deformation of carbon nanotubes in nanotube-polymer composites. Appl Phys Lett 74:3317-3319

15. Jin L, Bower C, Zhou O (1998) Alignment of carbon nanotubes in a polymer matrix by mechanical stretching. Appl Phys Lett 73:1197-1199

16. Sathyanarayana S, Hübner C (2013) Thermoplastic nanocomposites with carbon nanotubes. Struct Nanocompos 19-60

17. Ku BK, Emery MS, Maynard AD, Stolzenburg MR, McMurry PH (2006) In situ structure characterization of airborne carbon nanofibres by a tandem mobility-mass analysis. Nanotechnology 17:3613-3621

18. Wang H, Xu Z, Eres G (2006) Order in vertically aligned carbon nanotube arrays. Appl Phys Lett $88: 213111-213113$ 
19. Wu TM, Lin YW, Liao CS (2005) Preparation and characterization of polyaniline/multi-walled carbon nanotube composites. Carbon 43:734-740

20. Corcione CE, Frigione M (2012) Characterization of nanocomposites by thermal analysis. Materials 5:2960-2980

21. Gunasekaran SG, Devaraju S, Alagar M, Dharmendirakumar M (2014) Studies on synthesis and characterization of multi-walled carbon nanotube-reinforced polyimide nanocomposites based on a siloxane-modified anhydride. High Perform Polym 26:43-51

22. Yuen SM, Ma CCM, Chiang CL, Lin YY, Teng CC (2007) Preparation and morphological, electrical, and mechanical properties of polyimide-grafted MWCNT/polyimide composite. J Polym Sci Part A Polym Chem 45:3349-3358

23. Chang YH, Wu MS, Lin KF (2014) Graphting polyimide to MWCNT for enhancing dispersion and properties of MWCNT/polyetherimide nanocomposites. J Polym Res 21:419-425

24. Van Krevelen D (1975) Some basic aspects of flame resistance of polymeric materials. Polymer 16:615620

25. Johnson P (1974) A general correlation of the flammability of natural and synthetic polymers. J Appl Polym Sci 18:491-504 